\title{
Evolution de la qualité de l'énergie électrique
}

\author{
L. Berthet EDF R\&D, X. Mamo EDF R\&D, X. Yang EDF R\&D
}

\begin{abstract}
Ce document propose une vue générale de l'évolution du marché de l'électricité en France et de son impact sous l'angle de la qualité de l'électricité. Après quelques définitions préliminaires, un panorama du contexte réglementaire est proposé intégrant les dernières évolutions issues de la loi de programme fixant les orientations de la politique énergétique en France. Les aspects qualité de l'électricité contenus dans les contrats et référentiels de raccordement au réseau sont également abordés. Ces éléments montrent le rôle prépondérant donné aux opérateurs de réseaux de distribution, à la fois en terme de performance que de non discrimination. Une attention particulière est ensuite portée aux moyens mis en ouvre pour le suivi de la qualité par l'opérateur de réseau de distribution. Enfin, dans ce contexte de marché ouvert, les offres de conseil et de service proposées par le commercialisateur EDF sont présentées.
\end{abstract}

Index Terms - commercialisation, contrat, distribution, marché, normalisation, offre de service, performance, qualité de l’électricité, réglementation

\section{INTRODUCTION}

$\mathrm{L}^{\prime}$ Ouverture du marché de l'énergie a eu comme conséquence principale la séparation des différentes activités permettant de produire de l'énergie électrique et de l'acheminer jusqu'aux consommateurs. Les acteurs représentant ces activités existaient déjà, producteurs, opérateurs de réseau, commercialisateurs, mais leurs mission et prérogatives se sont vues assez nettement modifiées par ce nouveau contexte marquant une séparation claire entre le domaine régulé (réseaux) et dérégulé (vente d’énergie).

L’opérateur de réseau qui détient le monopole de la distribution ou du transport d'électricité sur la zone géographique que couvre son réseau, est en relation d'une part avec les commercialisateurs d'énergie et d'autre part, avec les clients consommateurs ou producteurs d'énergie.

Dans le contexte de la déréglementation du marché de l'énergie, les opérateurs de réseaux électriques sont soumis à des obligations légales en matière de qualité de service avec les utilisateurs de réseaux qu'ils soient producteurs, autres gestionnaires de réseaux, commercialisateurs ou consommateurs.

L. Berthet travaille à EDF R\&D au sein du département « Mesure et système d'information pour les réseaux électriques » (MIRE) dans le groupe « Qualité de l'électricité » en tant qu'ingénieur chercheur expert.

$\mathrm{X}$. Mamo est le responsable du groupe «Qualité de l'électricité » du département MIRE.

$\mathrm{X}$. Yang travaille en tant qu'ingénieur chercheur expert au sein du groupe «Qualité de l'électricité » du département MIRE.

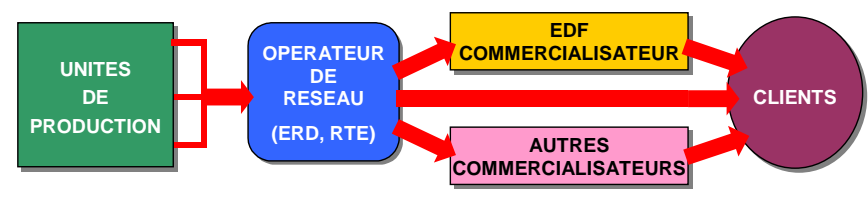

Fig 1 : Organisation des relations pour la fourniture d’électricité

Il convient de préciser ce que recouvre exactement la qualité de service qui est avant tout une appellation générique. Cette démarche n'est pas aisée et de nombreuses tentatives de classification des aspects qualitatifs de la qualité de l'énergie électrique ont été entreprises. Nous présentons ici les définitions prônées par le CEER (Council of European Enregy Regulators) et la CEI (Commission Electrotechnique Internationale). Ainsi la qualité de l'énergie électrique regroupe :

la qualité commerciale, de portée générale et visant à mesurer le service délivré par les fournisseurs et l'opérateur de réseau (temps de réponse aux questions des clients, rapidité d'intervention en cas de panne, temps de résolution des plaintes, rapidité de connexion au réseau, etc) ;

la qualité de l'électricité, qui englobe :

- la continuité de l'alimentation, relative aux interruptions de l'alimentation (coupures longues et brèves),

- la qualité de la tension, qui regroupe toutes les perturbations de l'onde électrique (creux de tension, fréquence, variations lentes de tension, harmoniques, déséquilibre, flicker) au sens de la norme EN50160.

\section{II.LE CONTEXTE REGLEMENTAIRE}

La libéralisation du marché de l'électricité a été amorcée par la directive européenne 96/92/CE du 19 décembre 1996 dont l'objectif est de mettre en place un marché intérieur de l'électricité. Elle a été abrogée par la directive 2003/54/CE du 26 juin 2003 concernant les règles communes pour le marché intérieur de l'électricité.

La transposition en législation française de cette directive est la loi 2000-108 du 10 février 2000 relative à la modernisation et au développement du service public de l'électricité, complétée en 2003. Cette loi indique que les activités de transport et de distribution, qui restent sous monopole, doivent être financièrement et comptablement séparées des activités de production et de commercialisation pour lesquelles la concurrence se met en place. La loi 2004803 du 9 août 2004 prévoit même une séparation juridique entre les activités de transport d'une part et les activités de 
production et de fourniture d'électricité d'autre part, qui devront être exercées par des personnes morales distinctes.

Un groupe d'étude spécial, référencé GS25, a été constitué afin de travailler sur le nouveau besoin défini dans les différentes lois citées. Il a pour objectif de publier un document définissant les niveaux de qualité qui doivent être fournis par les opérateurs de réseaux. Ce groupe réunit des représentants de chaque partie prenante des réseaux électriques: opérateur du réseau de distribution (ERD), opérateur du réseau de transport (RTE), gouvernement français, concessions, universités, etc.

La loi de programme 2005-781 du 13 juillet 2005 fixant les orientations de la politique énergétique intègre de part son article 60 une modification de la loi du 10 février 2000 (article 21) en ajoutant des dispositions relatives à la qualité de l'électricité. Il revient à la Commission de régulation de l'énergie et au Conseil supérieur de l'énergie de fixer par décret les niveaux et prescriptions techniques en matière de qualité que les opérateurs du réseau de transport et de distribution doivent respecter (niveaux pouvant être modulés par zones géographique). D’autre part, lorsque le niveau de qualité n'est pas atteint en matière d'interruptions d'alimentation imputables aux réseaux publics de distribution, le gestionnaire du réseau public de distribution concerné peut être obligé à remettre entre les mains d'un comptable public une somme qui sera restituée après constat du rétablissement du niveau de qualité.

\section{A. Contrats et engagements}

L’ouverture du marché a eu un impact important sur la contractualisation. En effet, il a fallu passer de contrats intégrés incluant acheminement et fourniture d'énergie à des contrats distinguant de manière explicite ces différentes volets. La structure contractuelle existante donne la possibilité aux clients d'être titulaires soit d'un contrat unique regroupant accès au réseau et fourniture soit de deux contrats distincts. Cette organisation est approuvée par le régulateur et toute modification doit être discutée par le CURDE (Comité des Utilisateurs du Réseau de Distribution Electrique).

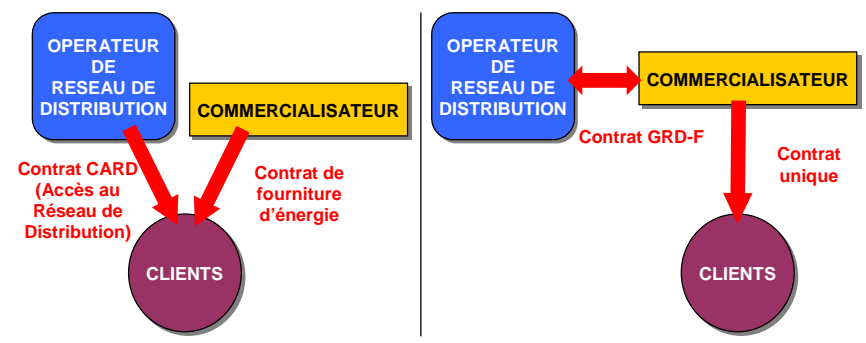

Fig. 2 : Structure contractuelle entre les différents acteurs

\section{1) En basse tension}

En terme de qualité de l'énergie, différentes dispositions existent en fonction du niveau de tension auquel les clients sont raccordés. Pour les clients en basse tension, deux décrets de loi existent et fixent les contraintes que le distributeur doit respecter, celles ci étant intégrées dans les contrats passés avec les clients sur ce segment :
- le décret du 29 mai 1986 qui fixe la plage admissible de variation de la tension nominale entre $207 \mathrm{~V}$ et 244 $\mathrm{V}(-10 \%,+6 \%)$,

- le décret du 26 avril 2001 qui porte sur les coupures de plus de 6 heures en indemnisant les clients coupés d'un montant de $2 \%$ de l'abonnement annuel.

\section{2) En moyenne tension}

En ce qui concerne les clients raccordés en moyenne tension, il n'existe pas pour l'instant de législation dédiée. Les contrats existant comportent cependant des engagements précis en terme de qualité de l'électricité [1].

a) Continuité de l'alimentation

Ainsi les coupures planifiées pour travaux sont distinguées de celles causées par des incidents. Pour les premières, le distributeur s'engage à mettre en place des consultations préliminaires, d'informer les clients de manière individuelle ou collective et de ne pas dépasser le seuil de 2 coupures par an, chacune d'une durée maximum de 4 heures. En ce qui concerne les incidents, la compensation pour toute coupure supérieure à 6 heures valorisée à $2 \%$ de l'abonnement annuel est présente tout comme pour la basse tension. Des engagements complémentaires sont définis sur le nombre maximum d'incidents en fonction du type de défaut (coupure brève ou longue) et de la zone géographique du site en excluant les catastrophes naturelles ou sabotages... En cas d'incident, il est également prévu de donner accès à tous les clients à un centre d'appel ouvert 24h/24 ainsi que d'envoyer un rapport d'incident à chacun d'entre eux au plus tard 2 jours après l'incident. Pour les clients de puissance supérieure à 2 MW, ils sont prévenus par téléphone au début et à la fin de la coupure et une analyse détaillée leur est envoyée au plus tard 1 mois après l'incident.

\begin{tabular}{|c|c|c|c|c|c|}
\cline { 3 - 6 } \multicolumn{1}{c|}{$\begin{array}{c}\text { Clients raccordés par } \\
\text { plusieurs alimentations } \\
\text { avec bascule automatique }\end{array}$} & $\begin{array}{c}\text { Coupures } \\
\text { (durée } \geq 1 \mathrm{~s})\end{array}$ & 36 & 13 & 6 & 4 \\
\hline \multirow{2}{*}{ Autres clients } & $\begin{array}{c}\text { Coupures longues } \\
\text { (durée } \geq 3 \text { min) }\end{array}$ & 6 & 3 & 3 & 2 \\
\cline { 2 - 7 } & $\begin{array}{c}\text { Coupures brèves } \\
(1 \mathrm{~s} \leq \text { durée < 3 min) }\end{array}$ & 30 & 10 & 3 & 2 \\
\hline
\end{tabular}

Fig. 3 : Nombre de coupures maximum par zones géographique

b) Qualité de la tension

Des engagements sont également pris sur la qualité de la tension, à savoir :

- Les variations de tension : la valeur contractuelle de la tension $\left(\mathrm{U}_{\mathrm{C}}\right)$ est située dans une plage de $\pm 5 \%$ autour de la tension nominale $\left(\mathrm{U}_{\mathrm{n}}\right)$. La valeur efficace de la tension de fourniture $\left(\mathrm{U}_{\mathrm{f}}\right)$ peut varier de $\pm 5 \%$ autour de la valeur contractuelle. Ces valeurs sont des moyennes 10 minutes.

- Les fluctuations rapides de la tension : le distributeur s’engage à ce qu'en permanence la sévérité du flicker ne dépasse pas le niveau de sévérité de longue durée, actuellement Plt $=1$. Les à-coups de tension sont compris dans cet engagement.

- Le déséquilibre : le taux moyen de déséquilibre ne doit 
pas dépasser la valeur de $2 \%$.

- La fréquence: en fonctionnement interconnecté par liaisons synchrones, la valeur de la fréquence varie dans la plage : $50 \mathrm{~Hz} \pm 1 \%$. Ces valeurs sont des moyennes 10 secondes.

A titre indicatif, des niveaux de tension harmoniques limites sont également précisés.

\begin{tabular}{|c|c|c|c|c|c|}
\hline \multicolumn{4}{|c|}{ Harmoniques impairs } & \multirow{2}{*}{\multicolumn{2}{|c|}{$\begin{array}{c}\text { Harmoniques } \\
\text { pairs }\end{array}$}} \\
\hline \multicolumn{2}{|c|}{ non multịles de 3} & \multicolumn{2}{|c|}{ multịples de 3} & & \\
\hline Rang & Seuils (\%) & Rang & Seuils (\%) & Rang & Seuils (\%) \\
\hline 5 & 6 & 3 & 5 & 2 & 2 \\
\hline 7 & 5 & 9 & 1,5 & 4 & 1 \\
\hline 11 & 3,5 & 15 et 21 & 0,5 & 6 à 24 & 0,5 \\
\hline 13 & 3 & & & & \\
\hline 17 & 2 & & & & \\
\hline 19,23 et 25 & 1,5 & & & & \\
\hline
\end{tabular}

Fig. 4 : Niveaux harmoniques limites pour la moyenne tension

Il est également prévu des engagements personnalisés liés aux conditions locales d'alimentation pouvant porter sur les coupures mais aussi sur les creux de tension de profondeur supérieure à 30\% et de durée supérieure à 600 ms.

\section{B. Raccordement au réseau}

La transposition de la directive sur l'ouverture des marchés de l'énergie a été accompagnée par la définition de règles techniques de raccordement au réseau sous la forme d'un décret 2003-229 et de deux arrêtés techniques, datés respectivement du 13 et 17 mars 2003. Ces derniers définissent les principes généraux mais pas leur mise en œuvre pratique, tâche que l'opérateur de réseau a repris à sa charge en mettant en place référentiels techniques, méthodes et outils d'étude.

Avant de réaliser le raccordement d'une unité de production ou de consommation, le distributeur entreprend des études en 3 niveaux - s'appuyant sur des normes et campagnes de mesures. L'objectif est d'anticiper les perturbations qui risquent d'être produites par l'installation à raccorder et de déterminer le point de raccordement le plus adapté. A travers cette démarche, il s'agit pour l’opérateur de réseau d'assurer un niveau de performance optimal pour tous les utilisateurs du réseau [2].

Le premier niveau consiste à identifier les caractéristiques de l'installation à raccorder et à faire une étude d'impact générique (capacité d'accueil, stabilité).

Le second niveau consiste à évaluer plus spécifiquement les perturbations induites par le raccordement, à savoir le flicker, le déséquilibre, les harmoniques et l'atténuation du signal tarifaire (TCFM : télécommande centralisée à fréquence musicale).

Le troisième niveau consiste à réaliser une étude détaillée de raccordement pour les perturbations identifiées. Dans le cas de l'éolien, celle ci repose sur la norme CEI 61400-21 qui permet d'évaluer l'impact en terme de qualité du raccordement.

\section{Normalisation}

La normalisation du domaine électrotechnique se décline à trois niveaux à savoir international avec la CEI (Commission Electrotechnique Internationale), européen avec le CENELEC (Comité Européen de Normalisation ELECtrotechnique) et national avec l'UTE (Union Technique de l'Electricité).

$\mathrm{Au}$ niveau international, le comité d'étude 77A est en charge des questions liées à la compatibilité électromagnétique. Différentes normes sont rattachées à ce comité :

- des normes d'environnement, qui définissent les niveaux de perturbation rarement dépassés sur les réseaux : CEI 61000-2-2 pour les réseaux publics basse tension et CEI 61000-2-12 pour la moyenne tension ;

- des normes d'émission et des rapports techniques qui définissent les niveaux de perturbation maximum pouvant être émis par des appareils raccordés au réseau public (cf. Fig 5) ;

- des normes d'immunité qui définissent les niveaux de perturbation minimum que les appareils peuvent supporter sans dégradation de leur performance ;

- des normes relatives aux méthodes de mesures : CEI 61000-4-7 pour les harmoniques, CEI 61000-4-15 pour le flicker, et CEI 61000-4-30 pour la qualité de la tension.

\begin{tabular}{|c|c|c|c|c|c|}
\cline { 2 - 6 } \multicolumn{2}{c|}{} & \multicolumn{3}{c|}{ BT } & \multirow{2}{*}{ MT et HT } \\
\cline { 2 - 6 } \multicolumn{2}{c|}{} & $<16 \mathrm{~A}$ & $16 \mathrm{~A}<<75 \mathrm{~A}$ & $>75 \mathrm{~A}$ & \\
\hline \multirow{2}{*}{ Harmoniques } & Norme & $61000-3-2$ & $61000-3-12$ & & \\
\cline { 2 - 6 } & Rapport & & & $61000-3-4$ & $61000-3-6$ \\
\hline \multirow{2}{*}{$\begin{array}{c}\text { Fluctuations } \\
\text { de tension }\end{array}$} & Norme & $\begin{array}{r}61000-3-3 \\
61000-3-11\end{array}$ & $61000-3-11$ & & \\
\cline { 2 - 6 } & Rapport & & & $61000-3-5$ & $61000-3-7$ \\
\hline
\end{tabular}

Fig. 5 : Normes d'émission CEI

Au niveau européen, deux éléments principaux relatifs au domaine sont à noter. Le document HD 472 S1 fixe les variations de tension admissibles sur les réseaux basse tension, à savoir $230+6 \%-10 \%$. La norme EN 50160 décrit quant à elle les caractéristiques admissibles du produit électricité pour les réseaux moyenne tension et basse tension hors circonstances exceptionnelles.

Seules les normes d'émission et le document HD 472 S1 sont harmonisés avec les directives européennes 73/23/CEE (basse tension) et 89/336/CEE (CEM).

\section{LA QUALITE DU COTE DU DISTRIBUTEUR}

\section{A. Organisation de la distribution}

La loi du 9 août 2004 a défini la nouvelle organisation du groupe EDF avec notamment l'indépendance de RTE et le partage des activités de distribution dont une partie est commune entre gaz et électricité. L’opérateur du réseau de distribution est constitué de deux entités complémentaires ERD (EDF Réseau Distribution) et EGD (EDF-GDF Distribution) et responsables ensemble de sa performance globale.

ERD définit, structure et gère les politiques en terme d'exploitation, de décision d'investissement et de gestion des 
actifs. Il négocie et co-signe les contrats de concession, et garantit la non discrimination et la transparence vis à vis des producteurs pour tout ce qui touche à l'accès au réseau. De plus, ERD est en relation avec les autorités de régulation en regard de ses différentes missions et est responsable de la gestion de la performance globale.

EGD est responsable quant à lui de l'exploitation, du développement et de la maintenance des actifs réseau. Sa mission consiste au quotidien en la bonne marche des réseaux, la gestion des matériels pour ce faire, la fourniture des services de comptage ainsi que les relations avec les autorités locales, les autorités concédantes et clients non éligibles.

\section{B. Indicateurs et dispositifs de suivi}

Afin de suivre et de mesurer l'évolution de la qualité de l'énergie, le distributeur calcule un certain nombre de critères sur la base de données réseau [3]. Ces indicateurs permettent notamment de statuer sur les besoins en terme d'amélioration des infrastructures. Ainsi, les valeurs suivantes sont définies :

- SAIDI, ou critère $B$ en France, indicateur de durée moyenne de coupure sur le réseau, pour les clients basse tension, 57 min en 2004 ;

- SAIDI pour les clients moyenne tension pondérée par la puissance fournie, 38 minutes en 2004 ;

- SAIFI, indicateur de nombre moyen de coupures sur le réseau, pour les coupures longues (de plus de 3 minutes), les coupures brèves (entre 1 seconde et 3 minutes) et les coupures très brèves (de moins de 1 seconde) ;

- Le nombre annuel de clients affectés par plus de 6 coupures longues, 30 coupures brèves, 70 coupures très brèves ou par plus de 3 heures d'interruption.

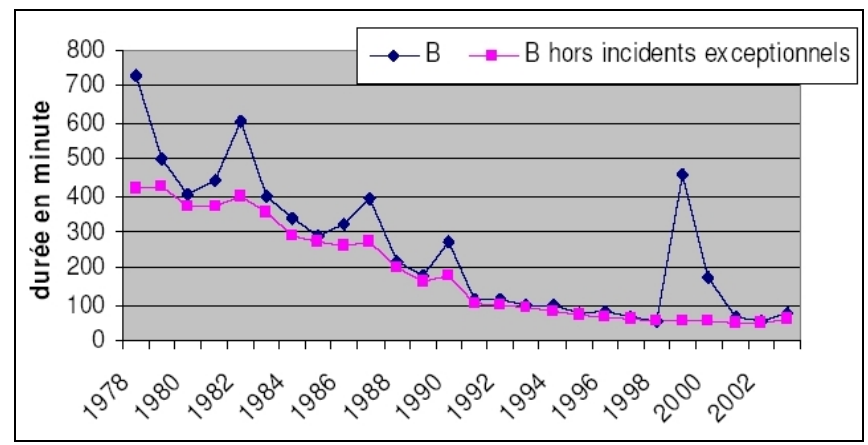

Fig. 6 : Evolution annuelle de l'indicateur de durée moyenne de coupure en basse tension (critère B)

Un dispositif de suivi des engagements contractuels est également en service [4] afin de pouvoir mesurer la qualité de la tension aux points de livraison des clients ayant souscrit le contrat selon les normes en vigueur (CEI 61000-4-30). Le système permet de traiter de manière automatique les données sur toute la chaîne de mesure afin de produire un rapport conforme à la norme EN50160 sur les niveaux de perturbation et les évènements constatés pendant la période considérée.

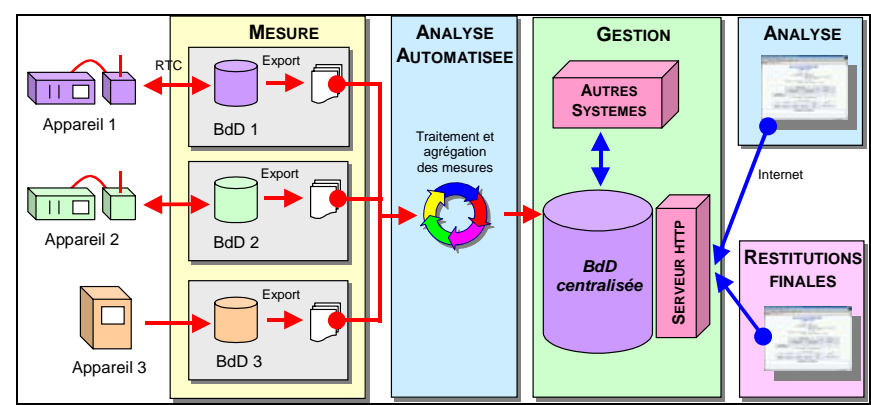

Fig. 7 : Dispositif de mesure pour le suivi des engagements contractuels

De même afin de suivre l'évolution dans le temps de paramètres de la qualité de la tension tels que les harmoniques, une campagne de mesure a été entreprise sur un échantillon d'une vingtaine de réseaux basse tension. En effet, les harmoniques augmentent du fait de la multiplication des charges non-linéaires. Or, il faut les maintenir en dessous des niveaux de compatibilité, afin que l'ensemble des équipements alimentés par les réseaux publics puissent fonctionner dans des conditions satisfaisantes.

Les premiers résultats obtenus en 2000 [5] [6] ont mis en évidence un accroissement moyen d'environ $1 \%$ en 10 ans sur l'harmonique de rang 5 .

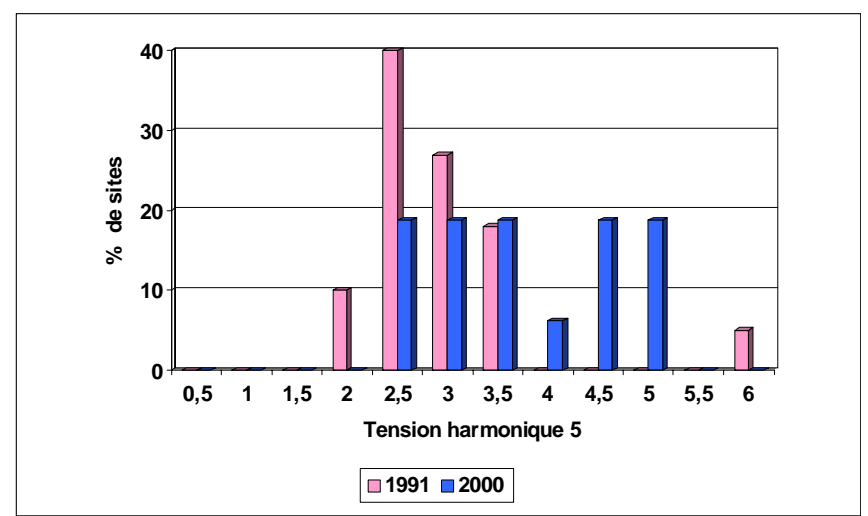

Fig. 8 : Evolution des niveaux de tension harmonique de rang 5 sur les réseaux BT entre 1991 et 2000

\section{LES OFFRES QUALITE DU COMMERCIALISATEUR EDF}

Pour les commercialisateurs de l'électricité, l'ouverture du marché est une opportunité pour proposer des offres de service et de conseil en complément de la fourniture électrique. De plus, la levée du principe de spécialité consécutive au changement de statut juridique d'EDF rend possible une plus grande diversité de telles offres. La Branche Commerce EDF a ainsi mis en place différentes gammes à destination des clients grandes entreprises et PME/PMI pour répondre à ce nouveau contexte. Il s’agit de :

- La gamme « OPTIMIA », axée sur la problématique de l'efficacité énergétique avec des offres de :

$>$ pré-diagnostic en industrie et tertiaire ;

$>$ conseil sur l'utilisation optimisée de l'éclairage pour les commerces et bureaux ;

$>$ conseil sur les usages électriques dans les bâtiments tertiaires dans le cadre de projets neuf ou rénovation. 
- La gamme «EQUILIBRE », axée sur du conseil pour l'environnement,

- la gamme «EXCELIS », axée sur la problématique de la qualité et de la fiabilité de l'alimentation électrique. Un réseau d'une trentaine d'experts technicocommerciaux dans ce domaine a été créé avec l’appui d'EDF R\&D pour la porter. Elle se décline en trois offres :

$>$ «Diagnostic Excelis » : il s’agit d'une étude de l'alimentation du client et de son process, lui permettant d'en acquérir une vision globale et reposant sur :

* l'identification des origines des perturbations qui affectent la fourniture d'électricité (phénomènes atmosphériques, moteurs, convertisseurs électroniques...) ;

* l'estimation des coûts de non-qualité : pertes de production, de données informatiques, casse ;

* la présentation de l'éventail des pistes de solutions possibles ;

* des recommandations techniques des solutions de désensibilisation.

$>$ «Performance Excelis »: faisant suite au volet précédent, cette offre consiste à une mise en place des équipements de désensibilisation pour fiabiliser la totalité ou une partie des équipements sensibles à la non qualité électrique. Il s'agit d’un contrat de qualité à la carte entre EDF et le client.

$>$ «Puissance Excelis » : il s'agit d'une offre visant à remplacer ou modifier le poste d'alimentation du client.

\section{V.CONCLUSION}

La législation Française intègre d'ores et déjà dans la loi de programme 2005-781 du 13 juillet 2005 fixant les orientations de la politique énergétique des dispositions relatives à la qualité de service et plus précisément la continuité de l'alimentation. Ces éléments constituent les premiers pas d'une régulation par la qualité, et il est à prévoir que d'autres phases se succèdent, chacune tenant compte du retour d'expérience de la précédente, avant d'arriver à un équilibre. Un autre élément important est l'échéance de 2007 qui verra l'ouverture totale du marché de l’électricité Français intégrant ainsi les clients résidentiels dans le secteur en concurrence.

Dans ce contexte de changements, il est indéniable que la situation telle qu'elle apparaît aujourd'hui risque d'être fortement modifiée pour les différents acteurs présents sur ce marché. Le distributeur devra prendre en compte les contraintes issues de la Commission de Régulation afin d'assurer un équilibre entre les coûts d'acheminement et les investissements et la maintenance à réaliser sur les réseaux pour maintenir des niveaux de qualité adaptés. Le commercialisateur devra quant à lui adapter ses offres au nouveaux segments de marché en intégrant des considérations d’efficacité énergétique de plus en plus présentes.

\section{REFERENCES}

[1] Contrat d'Accès au Réseau de Distribution, Contrat Emeraude

[2] C. Naslin, O. Gonbeau, JL. Fraisse, TC. Thai, "Wind farm integration power quality management for French distribution network", CIRED 2005.

[3] P. Cruchon, S. Sabeg, Y. Coatanea, N. Baumier-Duphil, "Power quality regulation and standardization, the French example", CIRED 2005.

[4] B. Paszkier, C. Santander, "IQS, a new format for the power quality data", CIRED 2005.

[5] L. Berthet, D. Boudou, X. Mamo, " Premiers résultats de la campagne de mesure des harmoniques sur les réseaux basse-tension francais", CIRED 2001.

[6] L. Berthet, D. Boudou, X. Mamo, P. Eyrolles, J. Martinon "STATE OF PLAY OF THE HARMONIC LEVELS ON THE FRENCH LOWVOLTAGE NETWORKS", CIRED 2003. 